\title{
An Unknown Force Awakened by A Pyramidal Structure
}

\section{Osamu Takagi1 , Masamichi Sakamoto², Hideo Yoichi' ${ }^{1}$, Hideyuki Kokubo1, Kimiko Kawano1, Mikio Yamamoto ${ }^{1}$}

\author{
${ }^{1}$ Information and Research Center, International Research Institute (IRI), 1108-2 Sonno, Inage, Chiba 263- \\ 0051, Japan \\ ${ }^{2}$ Aquavision Academy, 1228-3 Tsubuura, Narita, Chiba 287-0236, Japan
}

\begin{abstract}
We have been studying a non-contact effect by a meditator in a pyramidal structure on bio-sensors which are placed some distance from the meditator. So far we have demonstrated a meditator's non-contact effect with very high statistical accuracy and also discovered that the non-contact effect has a time dependence. However, the details of the experimental results were completely opposite our expectations. Namely, to our surprise, the noncontact effect was detected when the meditator was not in the pyramidal structure, and it was not detected when the meditator was in the pyramidal structure. In this paper, we conducted a verification experiment to clarify the factors that led to the unexpected experimental results; in particular, we wanted to identify the necessary conditions for the non-contact effect to come into play. The verification experiment consisted of four patterns in which the presence/absence of the meditator and the presence/absence of the pyramidal structure are the differences in the experimental conditions. Before the verification experiment, we evaluated eight possible results which were expected from the four patterns. From the results of the verification experiment, we verified that the non-contact effect on bio-sensors was undetectable in the meditation period, regardless of the presence or absence of the meditator and the pyramidal structure. Furthermore, we showed that the condition in which the meditator is in the pyramidal structure during the meditation period is the only condition for the non-contact effect to be detected after the meditation period. The results of the verification experiment further solidified the hypothesis that had been proposed in our previous paper about the origin of the non-contact effect, i.e., how it comes into play. The hypothesis is as follows: The meditator exerts a peculiar influence on the environment around him, which does not have a direct effect on the bio-sensors. However, this peculiar influence is transformed by the pyramidal structure, which then has an effect on the bio-sensors.
\end{abstract}

Keywords: meditator, pyramidal structure, non-contact effect, delayed effect, bio-sensor, cucumber, gas

\section{Introduction}

There exist numerous tales and books about the unknown function of a pyramidal structure (PS). While the study of the unknown function of the PS is historically and scientifically very interesting, little has been published that is scientifically trustworthy, and there are actually no academic papers that have shown experimental data with very high statistical accuracy except ours $[1,2]$.

Since 2005, the International Research Institute (IRI) has been conducting scientific research on the phenomenon which we refer to as the non-contact effect in which the subject (healer) affects biosensors which are placed at some distance from the subject (healer). Experiments regarding this phenomenon are referred to as healing experiments hereafter. We have developed various, completely new measuring methods using bio-sensors in order to detect the non-contact effect [3-5]. Using these measurement methods, we successfully detected the healer's non-contact effect on bio-sensors [6] and a wave like bio field around the healer [7].

The Sakamoto Hyper-tech Project (SHyP) in IRI was started in order to discover the unknown function of the PS and also to study its possible applications. In January 2010, the SHyP began the first experiment using the PS alone (without a meditator). In this experiment, we used bio-sensors (cucumbers) which had already given many satisfying results and the gas measuring method [4]. Bio-sensors were placed both on the top of the PS and at the Calibration Control Point (CCP) for $30 \mathrm{~min}$. The CCP was $8 \mathrm{~m}$ from the PS. Then the bio-sensors were separately placed within containers and stored to accumulate gas

This article is published under the terms of the Creative Commons Attribution License 4.0

Author(s) retain the copyright of this article. Publication rights with Alkhaer Publications.

Published at: http://www.ijsciences.com/pub/issue/2016-06/

DOI: 10.18483/ijSci.1038; Online ISSN: 2305-3925; Print ISSN: 2410-4477 
emitted from them. The gas concentrations were then measured for the sensors placed on the top of the PS and at the CCP, respectively. The idea of this experiment was that if a statistically significant difference was detected between the gas concentrations, we could conclude that the PS peak was indeed a peculiar point where an unknown energy or an unknown field may exist which had some effect on the bio-sensors.

However, as a result of measuring the gas concentrations of the PS peak and the CCP $(n=180)$, no significant difference was detected and we could not prove that the PS peak was a peculiar point.

On the other hand, there is an opinion that meditation within the PS has special effects on the meditator such as the mediator's healing capability [8]. If such an opinion is valid, there is a possibility that some interaction exists between the meditator and the PS. By taking this into account, in April 2011, we concluded the first experiment and moved into the second stage in which we started to investigate a combination of the PS and a meditator within it (PS meditation experiment). The experiment used the same gas measuring method as that employed in the first experiment.

Here, we describe the difference between healing experiments [6,7] and the PS meditation experiment. In the case of healing experiments, the healer concentrated his consciousness on the bio-sensors placed in front to affect the samples intentionally. In comparison, in the case of the PS meditation experiment, a meditator meditated without any directing attention to the bio-sensors placed overhead at the PS top. In addition, there are multiple numbers of subjects for the healing experiments, whereas the PS meditation experiment has just one.

We obtained two main results in our strictly carried out PS meditation experiment. As the first [1], we proved there was a very high statistical accuracy associated with the meditator's non-contact effect on the bio-sensors placed on the top of the PS. We used an analysis method that compared the "presence" ("the meditation period": Med) of a meditator with the "absence" ("after the meditation period": Post) of a meditator $\left(\mathrm{p}=3.13 \times 10^{-10}\right.$, t-test, two-tails $)$. As the second [2], we detected the non-contact effect over an extended period of time (more than 10 days) after the Post $\left(\mathrm{p}=3.51 \times 10^{-6}\right.$, Welch's t-test, two-tails). From this we proved a new phenomenon "an anomalous non-contact effect with a delay associated with a PS".

In this paper, we refer to the PS meditation experiment data we reported previously [2] as PS meditation experiment 2015 (PSME2015) to distinguish it from all other PS meditation experiments we have done since 2011.

When the PS meditation experiment study was started in 2011, we expected the following experimental results. The non-contact effect would be detected in the Med when the meditator is in the PS, while it would not be detected in the Post when the meditator is not in the PS. However, the experimental results turned out to be completely contrary to our expectations.

Among the experimental results of the PS meditation experiments, we focus here on the following two since they were opposite our expectations.

Experimental result 1: In the Med, when the meditator was in the PS, the non-contact effect was not detected.

Experimental result 2: In the Post, when the meditator was at least $5 \mathrm{~km}$ from the PS after the meditation, statistically significant data about the non-contact effect were detected.

The purpose of this paper is to understand the reasons why we obtained the unexpected results and to identify the necessary conditions for the non-contact effect to come into play. For this purpose, we reviewed the experimental conditions in detail and conducted a new verification experiment. From the results of the new verification experiment regarding Experimental result 1, we verified that the noncontact effect was not detected in the Med, regardless of the presence or absence of the meditator and the presence or absence of the PS. That is, having a meditator within the PS was not a necessary condition. Regarding Experimental result 2, we verified that the non-contact effect was statistically significant in the Post only under one set of the conditions, that is when a meditator was within the PS in the Med.

\section{Background of the experiment and the issue raised from the previous experiment}

To find the factor behind the unexpected result for the PS meditation experiment and to identify how the noncontact effect becomes effective, we conducted a new verification experiment, in which we varied the experiment conditions for PSME2015, i.e., the presence/absence of the meditator and the presence/absence of the PS. Before going into details of this experiment, we first explain the background of PSME2015 and the issue which became apparent through it. 


\section{2-1. PS Meditation Experiment 2015 (PSME2015)} [2]

The PSME2015 consisted of six experiment sets. Figure 1 shows one experiment set which was conducted over a two-day period. It consisted of the meditation period (Med) and the after-meditation period (Post). During the Med, a meditator performed 3 meditation runs starting at around 10:00 a.m. (med1 ), and performed another 3 runs after lunch starting at around 1:00 p.m. (med-2). Here, each meditation run lasted for $30 \mathrm{~min}$ (Hemi-Sync ${ }^{\circledR}$ [9]). After the Med, starting at about 3:00 p.m., 3 runs without a meditator (post-1) were conducted. Each lasted for 30 min. Finally, starting at about 6:00 a.m. the next day,
3 runs, $30 \mathrm{~min}$ for each and without a meditator, were conducted (post-2). In all runs, edible cucumber slices (Cucumis sativus 'white spine type') were used as bio-sensors; they were prepared following the simultaneous calibration technique (SCAT) [10] and were placed both on the PS peak and at the CCP $8 \mathrm{~m}$ from the PS. Immediately after each run, the biosensors were placed within containers and stored for about $36 \mathrm{~h}$ after which we measured the gas released. The intervals between experiment sets were at least 20 days because we had found the non-contact effect lasted for a long period after the Post, decreasing exponentially and finally reaching below the detection limit in about 20 days.

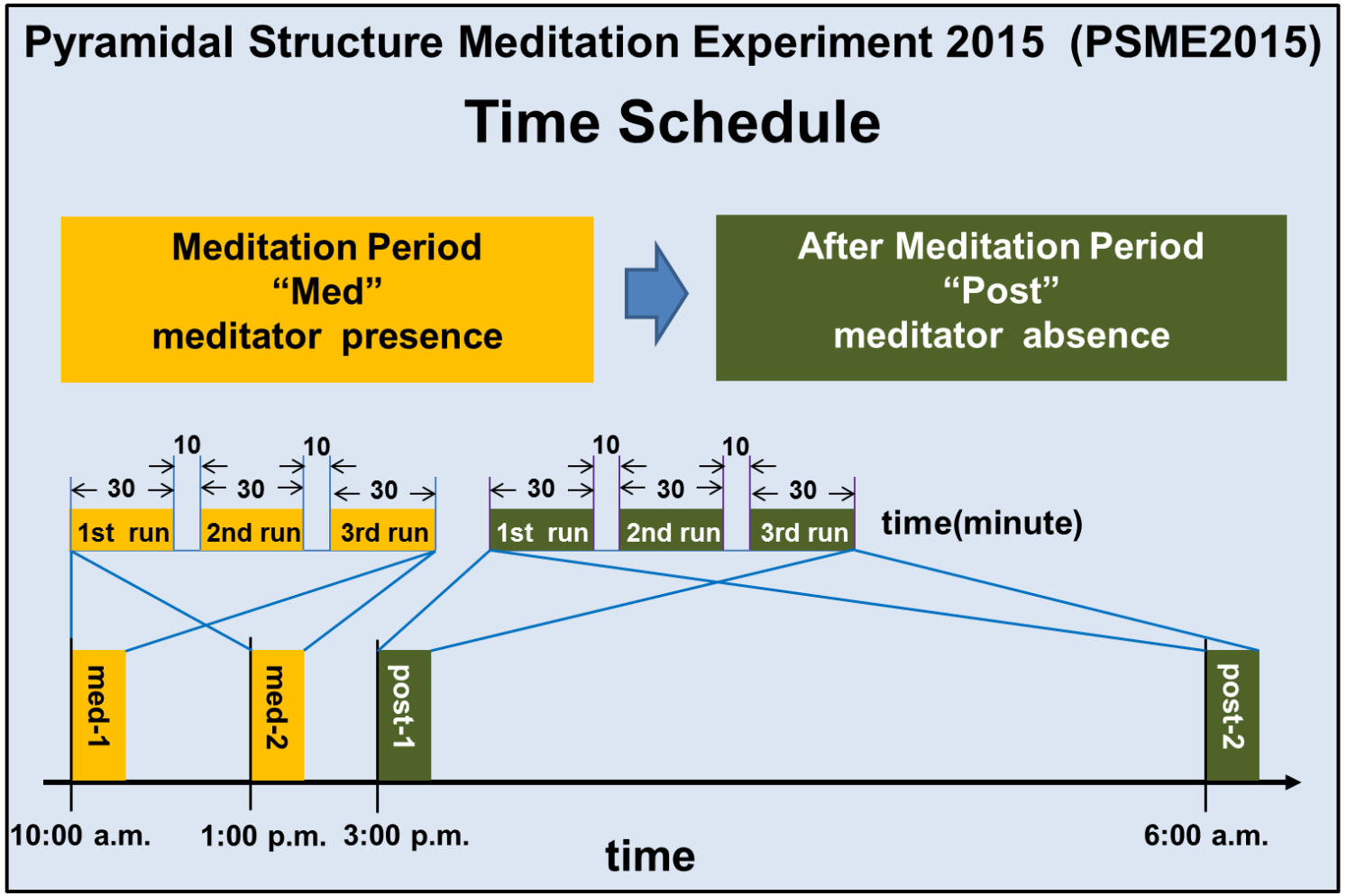

Fig. 1. The time schedule for the pyramid structure meditation experiment 2015 (PSME2015).

"The meditation period" (Med) is the period when a meditator meditates in the PS. "After the meditation period" (Post) is the period after the meditation when the meditator is at least $5 \mathrm{~km}$ from the PS. In the case of the Med, 3 meditation runs are conducted in the morning (med-1) and another 3 in the afternoon (med-2); each is 30 min long. In the case of the Post, 3 runs are conducted twice without a meditator (post-1, post-2).

\section{2-1-1. Pyramidal Structure (PS) [2]}

The PS employed in the PSME2015 and in the new verification experiment was the same. The PS frame was a square pyramid with a height of $107 \mathrm{~cm}$, a ridgeline length of $170 \mathrm{~cm}$ and a base length of 188 $\mathrm{cm}$. For a meditator to be able to go inside the PS, the base of the PS was raised at its corners by four tripods $73 \mathrm{~cm}$ from the floor. The four sides of the PS had Sierpinski triangle patterns consisting of aluminum plates. We called this pyramidal structure a fractal pyramid. Inside the PS, a transparent dome (85 cm diameter, $66.5 \mathrm{~cm}$ high) made of acrylic $(0.5$ $\mathrm{cm}$ thick) with a spherical shell shape was placed. The lower portion of the sphere had been removed where the diameter was $68.1 \mathrm{~cm}$. This allowed the meditator to insert his upper body inside the dome 
through the hole. The dome was designed in such a way that the meditator's voice resonated inside.

\section{2-1-2. Meditation method}

The meditator (an adult male with 23 years of HemiSync $^{\circledR}$ experience) was the same person who had participated in our previous reports, and he is one of the present authors (Masamichi Sakamoto). Regarding his participation in the meditation experiments, we had obtained his written consent. The meditation method in the Med of the PSME2015 was as follows. The meditator sat on a chair which was placed directly below the apex of the PS. At this time, the meditator's upper body was within the transparent acrylic dome inside the PS. He performed 3 meditation runs in the morning (med-1) and another 3 runs in the afternoon (med-2). During the meditation, he listened to Hemi-Sync ${ }^{\circledR}$, which is an audio technology designed to help the listener to meditate for an extended period of time. The meditator wore a set of stereo headphones so that there was no sound leak to the environment. He sometimes chanted. He meditated without directing any attention to the cucumber slices which were placed right above him. After the meditation run, he wrote down the content of the meditation. The meditation in the verification experiment followed the same procedure as above except for no PS.

\section{2-1-3. Gas measuring method}

In order to judge whether or not the non-contact effect exists, we adopted the gas measuring method which measures the gas concentration emitted from the edible cucumber slices which were kept in airtight containers for about $36 \mathrm{~h}$ after each run of the Med and the Post. The equipment used for the gas concentration measurement consisted of the gas sampling pump (GV-100, Gastech, Japan) and gas detecting tubes (141L, Gastech).

\section{2-1-4. Evaluation method of the non-contact effect (Calibration J value)}

In the PSME2015, the experimental samples (EXP1EXP4) and the control samples (CONT1-CONT4) were prepared by the SCAT. Here, (EXP1, CONT1), (EXP2, CONT2), (EXP3, CONT3) and (EXP4, CONT4) were pair samples. The cut plane of the pair samples of EXP and CONT shared the same plane. The experimental samples EXP1 and EXP2 were put in petri dishes placed on the PS peak, and EXP3, EXP4 and the control samples were put in petri dishes and placed at the CCP $8 \mathrm{~m}$ from the PS. After each run of the Med and the Post, EXP and CONT were kept in separate airtight containers and then the emitted 2-hexanol gas was measured simultaneously. To evaluate the power of the non-contact effect, we adopted the $\mathbf{J}$ value which is the natural logarithm of the ratio of the gas concentrations of EXP and
CONT.

$$
J=k \ln \left(\frac{C_{E X P}}{C_{C O N T}}\right) \quad \cdots(1)
$$

Here, $\mathrm{k}$ is a proportionality coefficient $(\mathrm{k}=1)$ [7]. $\mathrm{C}_{\text {EXP }}$ and $\mathrm{C}_{\mathrm{CONT}}$ are the gas concentrations in a container containing EXP and CONT. $\mathrm{J}_{\mathrm{E}}$ was calculated from the main experimental pair samples (EXP1, CONT1) and (EXP2, CONT2), and $\mathrm{J}_{\mathrm{CAL}}$ was the average of $\mathrm{J}_{\mathrm{E}}$ values calculated from the calibration experimental pair samples (EXP3, CONT3) and (EXP4, CONT4). The calibration value which deducted $\mathrm{J}_{\mathrm{CAL}}$ from $\mathrm{J}_{\mathrm{E}}$ expresses the power of the non-contact effect. To analyze the experiment result, we used the calibration value $\mathrm{J}_{\mathrm{E}-\mathrm{CAL}}$.

$$
J_{E-C A L}=J_{E}-J_{C A L} \quad \cdots(2)
$$

The blank experiment (number of samples $n \geq 12$ ) in the verification experiment was carried out in the state where neither a meditator nor the PS was present, and the standard deviation of $\mathrm{J}_{\mathrm{E}-\mathrm{CAL}}$ was 0.1 or less. Therefore, in this paper, we judge whether or not the non-contact effect to the bio-sensors was detected based on whether equation (3) was satisfied or not.

$$
\left|J_{E-C A L}\right| \geq 0.1 \quad \cdots(3)
$$

\section{2-2. Issue raised from the previous experiment}

In the first experiment done for the SHyP, only the PS was employed without a meditator in order to study the unknown function of the PS. However, we were not able to detect the unknown function of the PS. Next, we started the PS meditation experiment with a meditator sitting inside the PS in order to detect the non-contact effect. At the start of the PS meditation experiment, we anticipated that the noncontact effect would be detected in the Med in which the meditator is in the PS. Also we anticipated that the non-contact effect would not be detected in the Post because the meditator was not in the PS and was at least $5 \mathrm{~km}$ from it. From the results of the healing experiments, we thought that the non-contact effect to bio-sensors was a real-time effect exerted by the subject (meditator). Therefore, in the PS meditation experiment, we anticipated that the meditator would exert a real-time non-contact effect on the bio-sensors placed on top of the PS; thus, when there was no meditator in the PS, there would be no non-contact effect on the bio-sensors.

However, for the PS meditation experiment, no noncontact effect was detected in the Med during which 
the meditator was in the PS. We attributed this to $\mathrm{J}_{\mathrm{E}-}$ $\mathrm{CAL}$ of the Med becoming zero within the margin of error and $\left|\mathrm{J}_{\mathrm{E}-\mathrm{CAL}}\right|<0.1$ was satisfied. In contrast, we detected the non-contact effect in the Post in which the meditator was not in the PS. We attributed this to the statistically significant difference of $\mathrm{J}_{\mathrm{E}-\mathrm{CAL}}$ being detected between the Med and the Post and $\mathrm{J}_{\mathrm{E}-\mathrm{CAL}}$ of the Post satisfied $\left|\mathrm{J}_{\mathrm{E}-\mathrm{CAL}}\right| \geq 0.1$ [11]. These results of the PS meditation experiment were surprising and were completely contrary to our anticipation. In order to understand the factors that led to these unexpected results, we took an analytical approach. The main contributing factors to the result of the Med were as follows: the meditator, the PS, the combination of the meditator and the PS, and conditions that were due to neither the meditator nor the PS. To identify the factors that lead to the experimental results of the Med and the Post, we conducted a verification experiment.

\section{Verification experiment \\ 3-1. Analytical approach}

From the PSME2015, we thought the main variables of the verification experiment were the meditator and the PS. To identify the prime factor for the unexpected experimental results of the Med and the Post, the presence of the meditator and the PS were considered as the condition variables in the Med. As a result, the verification experiment for the Med would have the four patterns shown as (a)-(d) in Table 1.
Details of the experiment patterns (a)-(d) are as follows.

(i) Pattern (a): This was the pattern used in PSME2015. The meditator meditated in the PS and the bio-sensors which were physically separated from him were placed both on the PS peak and at the CCP $8 \mathrm{~m}$ from the PS.

(ii) Pattern (b): This was a verification experiment in which the bio-sensors were placed above the meditator's head without the PS. The height of the bio-sensors was the same as (a). Bio-sensors were also placed at the CCP $8 \mathrm{~m}$ from the PS.

(iii) Pattern (c): This was a verification experiment in which the bio-sensors were placed on the PS peak and the meditator was absent. Bio-sensors were also placed at the CCP $8 \mathrm{~m}$ from the PS.

(iv) Pattern (d): This was a verification experiment in which the bio-sensors were placed at the same height as (a) and both the meditator and the PS were absent. Bio-sensors were also placed at the CCP $8 \mathrm{~m}$ from the PS.

The Post was conducted after the Med without the meditator. Therefore, the Post had four patterns of (a')-(d'), each of which corresponded to (a)-(d) of the Med (Table 1). The sequence for the Med and the Post of the verification experiment was the same as that for the PSME2015 shown in Fig. 1. Figure 2 shows the experiment patterns for the Med and the Post.

Table 1 The experiment patterns for the Med and the Post.

The four patterns, shown as (a)-(d) and (a')-(d') are distinguished by the presence/absence of the meditator and the PS. The absence is indicated by an overbar above the words. 


\begin{tabular}{|c|c|c|c|}
\hline \multirow{2}{*}{\multicolumn{2}{|c|}{ Med }} & \multicolumn{2}{|c|}{ Pyramidal Structure (PS) } \\
\hline & & presence & absence \\
\hline \multirow{4}{*}{ 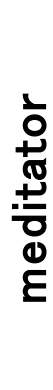 } & $\underset{0}{0}$ & (a) & (b) \\
\hline & $\begin{array}{l}0 \\
0 \\
0 \\
\vdots \\
0\end{array}$ & (Meditator AND PS) & $($ Meditator AND $\overline{P S})$ \\
\hline & $\stackrel{0}{0}$ & (c) & (d) \\
\hline & 茴 & $(\overline{\text { Meditator }}$ AND $P S)$ & $(\overline{\text { Meditator }}$ AND $\overline{P S})$ \\
\hline \multirow{2}{*}{\multicolumn{2}{|c|}{ Post }} & \multicolumn{2}{|c|}{ Pyramidal Structure (PS) } \\
\hline & & presence & absence \\
\hline \multirow{4}{*}{ 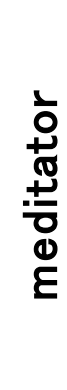 } & \multirow{4}{*}{ 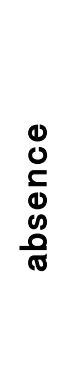 } & (a') & (b') \\
\hline & & $(\mathrm{a}) \rightarrow(\overline{\text { Meditator }} \quad A N D \quad P S)$ & (b) $\rightarrow(\overline{\text { Meditator }}$ AND $\overline{P S})$ \\
\hline & & $\left(c^{\prime}\right)$ & $\left(d^{\prime}\right)$ \\
\hline & & (c) $\rightarrow(\overline{\text { Meditator }}$ AND $P S)$ & $(\mathrm{d}) \rightarrow(\overline{\text { Meditator }}$ AND $\overline{P S})$ \\
\hline
\end{tabular}

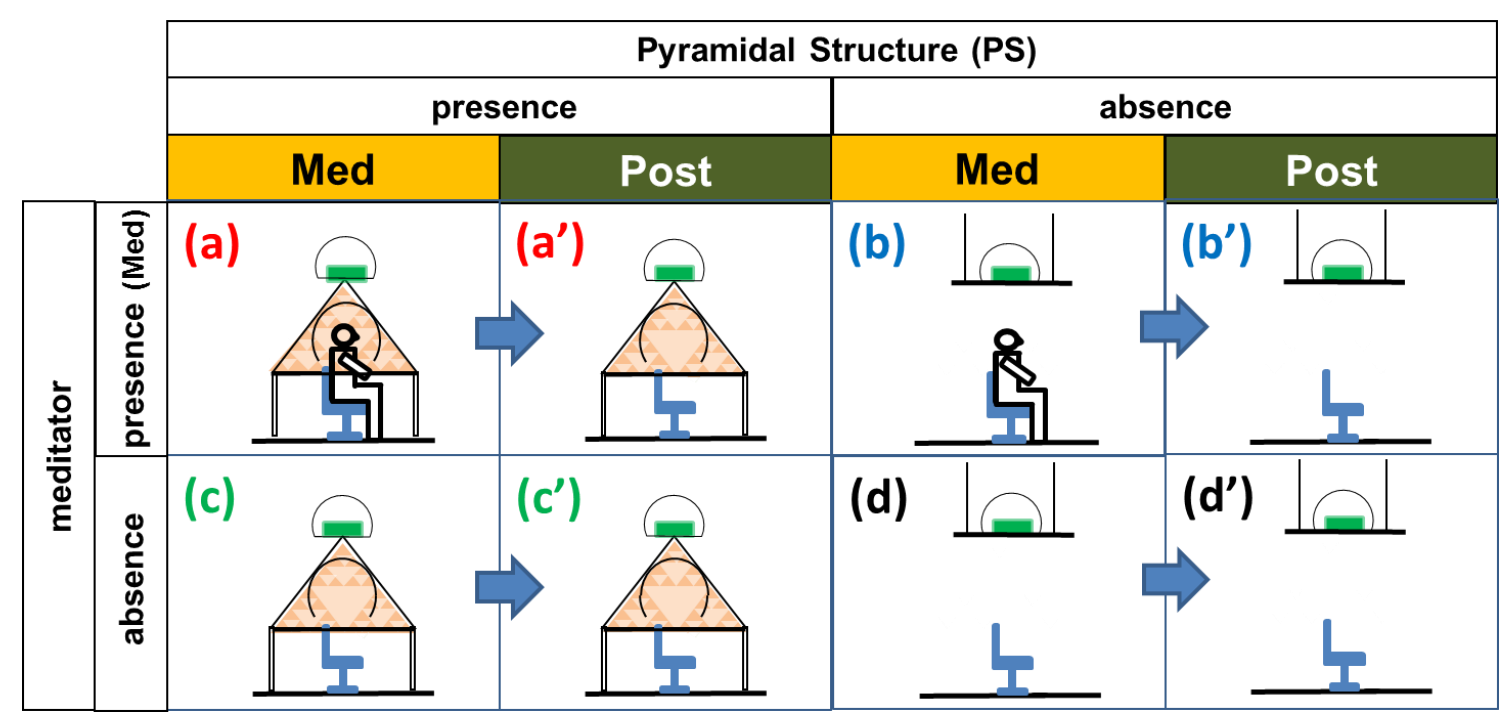

Fig. 2. The experiment patterns in Table 1 are illustrated.

3-2. Possibilities of certain results for the verification experiment and their interpretation

Table 2 shows the result possibilities of the four experiment patterns (a)-(d) for the Med and shows those of the four experiment patterns $\left(a^{\prime}\right)-\left(d^{\prime}\right)$ for the Post.

From four experiment patterns (a)-(d) for the Med, there are eight possible results (1)-(8). In Table 2, "0" shows the case in which the non-contact effect cannot be detected, that is, $\mathrm{J}_{\mathrm{E}-\mathrm{CAL}}$ becomes zero within the margin of error and $\left|\mathrm{J}_{\mathrm{E}-\mathrm{CAL}}\right|<0.1$ is satisfied. In addition, "1" shows the case in which the non-contact effect can be detected with statistical significance and $\mid \mathrm{J}_{\mathrm{E}-\mathrm{CAL}} \quad \geq 0.1$ is satisfied. In the introduction, we showed that the non-contact effect was not detected in Med in the PS meditation experiment 
(Experimental result 1). Therefore, all the result possibilities of pattern (a) in Table 2 were set to " 0 ". Since the Post was conducted after the Med without the meditator, the experiment patterns for the Post are $\left(a^{\prime}\right)-\left(d^{\prime}\right)$ and there are eight expected results $\left(1^{\prime}\right)-\left(8^{\prime}\right)$.
In the introduction, we showed that the non-contact effect was detected in the Post (Experimental result 2). Therefore, all the result possibilities for pattern (a') in Table 2 were set to " $1 "$.

Table 2 The result possibilities for the Med and Post.

Result possibilities (1)-(8) for four experiment patterns (a)-(d) and result possibilities (1')-(8') for four experiment patterns (a')-(d'). "0" shows the case in which the non-contact effect cannot be detected, while " 1 " shows the case in which the non-contact effect can be detected with statistical significance.

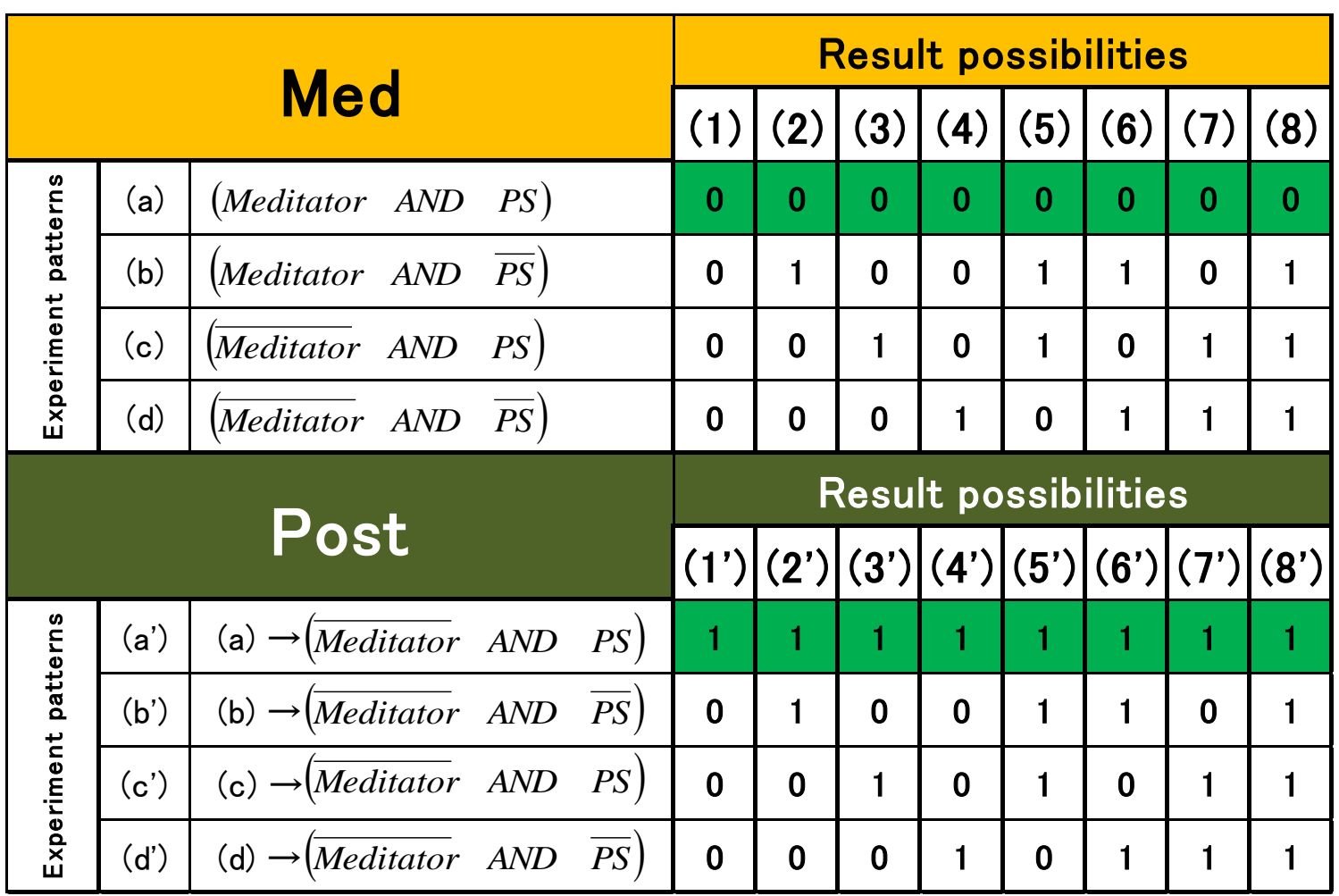

Next, we tried to understand the physical meanings of the eight result possibilities for the Med and the Post. From the first PS meditation experiment, our expectation that the non-contact effect would be detected in the Med was not met. From this, we understood that from the result possibilities in the Med we should aim at understanding the reason and identifying the factor behind the fact that the noncontact effect was not detected in the Med. On the other hand, understanding the result possibilities in the Post should aim at identifying the factor behind the fact that the non-contact effect was detected contrary to our expectation.

(I) Interpretation of the possible results for the Med in Table 2

(I -i) Result (1), (a,b,c,d) $=(0,0,0,0)$.

This result is the case when the non-contact effect is not detected, i.e., the non-contact effect in the Med becomes zero within the margin of error, regardless of the presence or absence of the meditator and the PS. In this case, the result possibilities for (c) and (d) being " 0 " are within the expectation since the meditator is not present, regardless of the presence or absence of the PS. However, the result possibilities for (a) and (b), in which the meditator is present, being " 0 " are beyond the expectation. This result implies that although a certain non-contact influence may exist around the meditator, the non-contact action does not have a real-time influence on the biosensors so that the non-contact effect may not be detected.

(I-ii) Result (2), (a,b,c,d) $=(0,1,0,0)$.

This result is the case for which the non-contact effect is detected only when the PS is absent and the meditator is present. In the result possibilities for (c) and (d) in which the meditator is absent, a peculiar effect not being detected is within the expectation. However, in the result possibilities for (a) and (b) in 
which the meditator is present, a peculiar effect is detected only for (b) in which the PS is absent. In this case, the meditator's non-contact influence may have a direct effect on the bio-sensors, and the PS may suppress it.

(I -iii) Result (4), (a,b,c,d) $=(0,0,0,1)$.

This result is the case when the non-contact effect is detected only when neither the meditator nor the PS is present. In the result possibility for (d), in which neither the meditator nor the PS is present, the effect being detected is beyond the expectation. The result possibility for (c) may imply that the PS might suppress the effect. Also, the result possibility for (b) may imply that the meditator might suppress the effect. Therefore, this result requires us to consider an adventurous possibility that the peculiar effect exists constantly around the samples, when neither the meditator nor the PS is present.

(I -iv) Result (6), (a,b,c,d) $=(0,1,0,1)$.

This result is the case for which the non-contact effect is detected when the PS is absent, regardless of the presence or absence of the meditator. In the result possibilities for (a) and (c), since the non-contact effect is not detected, the PS might suppress the effect. This result requires us to consider a possibility that the peculiar effect exists constantly around the samples, when the PS is absent, regardless of the presence or absence of the meditator.

(I -v) Result (7), (a,b,c,d) $=(0,0,1,1)$.

This result is the case for which the non-contact effect is detected when the meditator is absent, regardless of the presence or absence of the PS. Although it suggests a possibility that the peculiar effect exists constantly around the samples, (a) and (b) may imply that the meditator might suppress it. (I -vi) Result (8), (a,b,c,d) $=(0,1,1,1)$.

This result is the case for which the non-contact effect is not detected only when the meditator is present in the PS. This result may imply that the meditator in the PS might suppress the peculiar effect and it is necessary to consider a possibility that the peculiar effect exists constantly around the samples. (I-vii) Results (3) and (5), (a,b,c,d) $=(0,0,1,0)$, $(0,1,1,0)$.

It is difficult to explain these results by a simple theory. It seems that the relation between the meditator and the PS is quite complicated or involves other factors.

(II) Interpretation of the possible results for the Post in Table 2

(II-i) Result (1'), (a', b', c', d') $=(1,0,0,0)$.

This result is the case for which the non-contact effect is detected with statistical significance in the Post only when the meditator is present in the PS in the Med and is not detected otherwise. The result possibility for (a') shows the possibility that the meditator exerts a peculiar influence on the PS, which then transforms it into something else and emits it outside. Also, the result possibility for ( $b^{\prime}$ ) shows the possibility that the peculiar effect by the meditator does not have an effect on the bio-sensors when the PS is absent. It is necessary to consider the possibility that the relation between the meditator and the PS is the prime factor for the non-contact effect.

(II-ii) Result (2'), (a', b',c',d') $=(1,1,0,0)$.

This result is the case for which the non-contact effect is detected with statistical significance in the Post when the meditator is present in the Med, regardless of the presence or absence of the PS. This means the meditator is the prime factor for the noncontact effect and the PS is not. A peculiar residual effect may remain over the head of the meditator.

(II-iii) Result (3'), (a',b',c', d')=(1,0,1,0).

This result is the case for which the non-contact effect is detected with statistical significance in the Post when the PS is present in the Med, regardless of the presence or absence of the meditator. This shows a possibility that the PS itself holds the peculiar effect and the meditator does not.

(II-iv) Result ( $\left.8^{\prime}\right), \quad\left(a^{\prime}, b^{\prime}, c^{\prime}, d^{\prime}\right)=(1,1,1,1)$.

This result is the case for which the non-contact effect is detected with statistical significance in the Post, regardless of the presence or absence of the meditator and the PS. This means both the meditator and the PS are not the prime factors for the noncontact effect in the Post. Such a result shows a possibility that the cucumber activity changes with the measurement time and the setting position, for example. In this case, the difference in the place may not be the cause of the difference. Rather, there is a possibility that a delicate environmental difference, such as temperature or illumination, may influence the cucumber activity.

(II-v) Results (4'), (5'), (6'), (7'), $\left(a^{\prime}, b^{\prime}, c^{\prime}, d^{\prime}\right)=(1,0,0,1),(1,1,1,0),(1,1,0,1),(1,0,1,1$,$) .$

It is difficult to explain these results by a simple theory. It seems that the relation between the meditator and the PS is quite complicated or involves other factors.

\section{3-3. Setting up working hypotheses}

From the two experimental results of the PS meditation experiment described in the Introduction, we found the non-contact effect had a delay. We proposed one hypothesis that qualitatively explains the non-contact effect accompanied by a delay. The 
hypothesis is as follows: The meditator exerts a peculiar influence on the environment around him, which does not have a direct effect on the biosensors. This peculiar influence is transformed by the PS, which then has an effect on the bio-sensors.

However, the two experimental results mentioned above from PSME2015 are the only pieces of evidence for this hypothesis, which we believe are insufficient. Thus, in order to get further evidence, we must first look at the result possibilities for the verification experiment to see if there are any that justify the hypothesis.

We analyze which result possibilities out of the eight in Table 2 (1)-(8) and (1')-(8') for the Med and the Post, respectively, are the ones that can further justify the hypothesis.

(i) For the Med (Table 2)

Among the eight possibilities in Table 2, (1) can justify the first half of the hypothesis. The first half of the hypothesis is as follows: The meditator exerts an influence on the environment around him, which does not have a real-time effect on the bio-sensors. This would require the possible result value for the experiment patterns in which the meditator is present, i.e. (a) and (b), to be " 0 ". This would correspond to result possibilities (1)-(8) for experiment pattern (a) and (1), (3), (4) and (7) for pattern (b). For the experiment patterns in which the meditator is absent, i.e. patterns (c) and (d), it would be difficult to expect whether or not an effect on the bio-sensors exists since the presence of the meditator itself is denied. However, the result for the PS experiment without a meditator would suggest that the possible result value for (c) would be "0", which would correspond to possibilities (1), (2), (4) and (6). We could also infer that the possible result value for $(\mathrm{d})$ being " 1 " would be unlikely from section 3-2 (I-iii) and (I-iv). Thus the possible result value for (d) would be " 0 ", which would correspond to result possibilities (1) and (2). Therefore, we could conclude that the result possibility that can satisfy all the experiment patterns from (a) to (d) at the same time would be (1) only. (ii) In the Post (Table 2)

Among the eight possibilities in Table 2, (1') is the one that can justify the second half of the hypothesis. The second half of the hypothesis is as follows: This meditator's influence is transformed by the PS, which then has an effect on the bio-sensors. This would require that the possible result value for the experiment pattern in which both the meditator and the PS are present, i.e. (a') would be " 1 ", which would correspond to result possibilities (1')-(8'). For pattern (b'), in which the meditator is present while the PS is absent in Med, it would require the possible result value to be " 0 ", which would correspond to result possibilities (1'), (3'), (4') and (7'). For the experiment patterns in which the meditator is absent, i.e. (c') and (d'), it would be difficult to expect whether or not an effect on the bio-sensors exists since the presence of the meditator itself is denied. However, the result for the PS experiment without a meditator would suggest that the possible result value for (c') would be " 0 ", which would correspond to possibilities (1'), (2'), (4') and (6'). We could also infer that the possible result value for (d) being " 1 " would be unlikely from section 3-2 (II-iv). Thus the possible result value for (d') would be " 0 ", which would correspond to result possibilities (1') and (2'). Therefore, we could conclude that the result possibility that can satisfy all the experiment patterns from (a') to (d') at the same time would be (1') only.

Thus, we set up the following two working hypotheses and verify them. Doing so will identify the factors behind the two unexpected experimental results in the PS meditation experiment and also further justify the hypothesis that was proposed to explain how the non-contact effect with a delay comes into play.

\section{Working Hypothesis 1 (hypothesis about the Med):}

This is the working hypothesis "The reason the noncontact effect was not detected in (a) was not because experiment pattern (a) for the Med in Table 1 is special, but rather in all verification experiment patterns (b)-(d), the non-contact effect was not detected." In other words, it is a hypothesis that the non-contact effect is zero at the $99 \%$ confidence interval and $\left|\mathrm{J}_{\mathrm{E}-\mathrm{CAL}}\right|<0.1$ in all experimental patterns (a)-(d).

Working Hypothesis 2 (hypothesis about the Post): This is the working hypothesis "When the experiment pattern for the Post in Table 1 is (a'), the non-contact effect is detected with statistical significance of $\mathrm{p}<1 \%$, and $\left|\mathrm{J}_{\mathrm{E}-\mathrm{CAL}}\right| \geq 0.1$. Furthermore, as for other verification experiment patterns $\left(b^{\prime}\right)-\left(d^{\prime}\right)$, the peculiar effect is not detected."

The verification of the two Working Hypotheses can result in verifying result possibility (1) for the Med and result possibility (1') for the Post out of the eight result possibilities.

\section{Experiment Results}

Figure 3 shows the results of the four experiment patterns shown in Table 1. The horizontal axis shows time, whereas the vertical axis shows $\mathrm{J}_{\mathrm{E}-\mathrm{CAL}}$ which is an index for the strength of the non-contact effect. In Fig. 3, four measurement points are plotted on each of the four lines and they show the average time when the bio-sensors were placed at the PS peak in 
the 1 st run of med-1, med-2, post- 1 , and post- 2 . The times of the four points are about 10:00 a.m., 1:00 p.m., 3:00 p.m. and 6:00 a.m., respectively.

Figure 4 plots the average of med- 1 and med- 2 as the Med, and the average of post- 1 and post- 2 as the Post. In all experiment patterns (a)-(d), the Med showed that $\mathrm{J}_{\mathrm{E}-\mathrm{CAL}}$ was zero for the $99 \%$ confidence interval and $\left|\mathrm{J}_{\mathrm{E}-\mathrm{CAL}}\right|<0.1$. Also, the result of multiway analysis of variable (multi-way ANOVA) was $\mathrm{p}=0.517$. On the other hand, the Post showed that $\mathrm{J}_{\mathrm{E}-}$
CAL was zero for the $99 \%$ confidence interval and $\mid J_{E-}$ CAL $\mid<0.1$ in the experiment patterns $\left(b^{\prime}\right)-\left(d^{\prime}\right)$.

However, in the Post, $\mathrm{J}_{\mathrm{E}-\mathrm{CAL}}$ of the experiment pattern (a') had a statistically significant difference compared with ( $\left.b^{\prime}\right)-\left(d^{\prime}\right)$, and $\left|J_{E-C A L}\right| \geq 0.1$. Here, in the Post, the multi-way ANOVA was $\mathrm{p}=2.19 \times 10^{-4}$. From the above, result possibilities (1) and (1') were verified out of the eight result possibilities for the Med and the Post in Table 2.

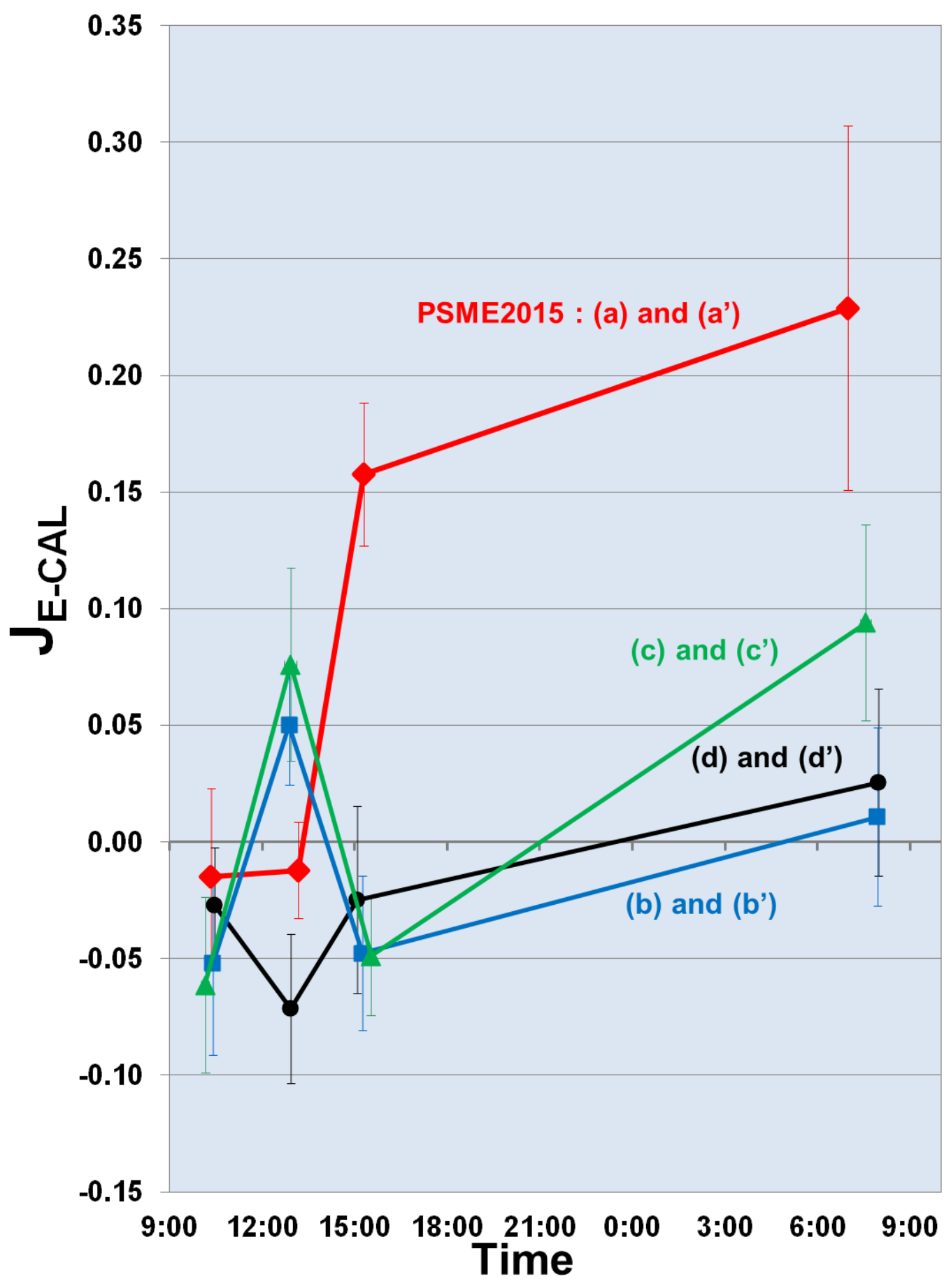


Fig. 3. The results of the PSME2015 and the verification experiment.

(a)-(a') is the results of the PSME2015. (b)-(b'), (c)-(c') and (d)-(d') are the results of the verification experiment. The horizontal axis is time. The vertical axis is $\mathrm{J}_{\mathrm{E}-\mathrm{CAL}}$ showing the strength of the non-contact effect. One line has four measurement points, each corresponding to the result for med-1, med-2, post-1 and post-2 from the left, respectively. The error bar shows standard error.

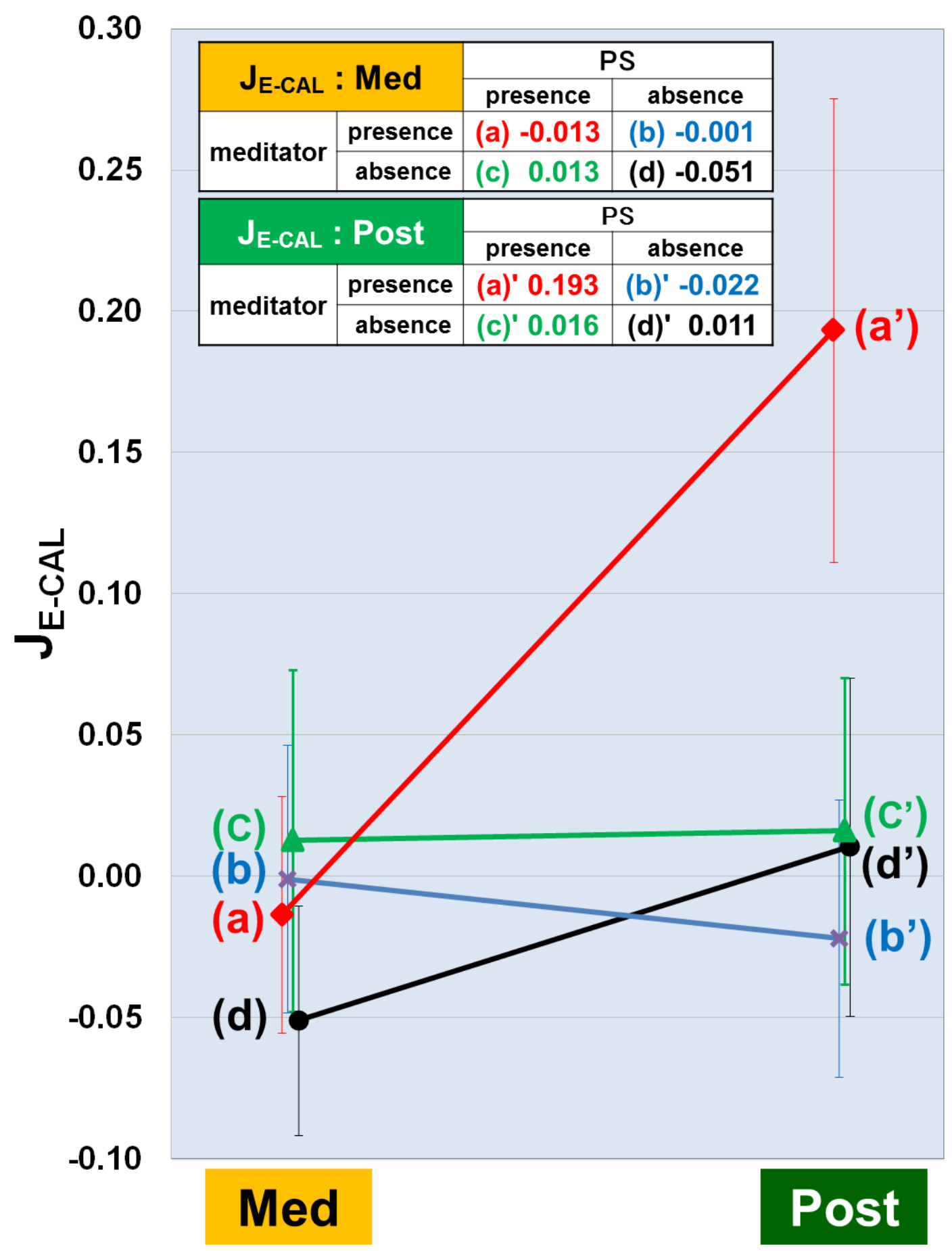

Fig. 4. The results of the PSME2015 and the verification experiment.

From the results of Fig. 3, Med shows the average of med-1 and med-2 and the Post shows the average of post- 1 and post-2. The vertical axis is $\mathrm{J}_{\mathrm{E}-\mathrm{CAL}}$ showing the strength of the non-contact effect. The error bar shows $95 \%$ confidence interval. To avoid overlapping, the error bars are shifted a little bit in the horizontal direction. The value of each point is shown in the table in the figure. 


\section{Conclusion and consideration}

The Working Hypothesis 1 and $\mathbf{2}$ have been verified with very high statistical accuracy as a result of the PSME2015 and the verification experiment described in this paper.

By verifying the Working Hypothesis 1, we verified that the reason the non-contact effect was not detected when the meditator was in the PS in the Med was not because it was a special condition. Instead, we verified that the non-contact effect was not detected in the Med, regardless of the presence or absence of the meditator and the PS. This result suggests that the direct effect on the bio-sensors does not exist in the Med, regardless of the presence or absence of the meditator and the PS. This result differs from that for the healing experiment. In neither the PS meditation experiment nor the verification experiment, did the meditator directed his attention to the bio-sensors. This condition may be the reason for this difference. We would probably need to conduct the PS meditation experiment in which the meditator directs attention to the biosensors during meditation. By verifying the Working Hypothesis 2, we verified that the non-contact effect was detected with statistical significance in the Post only in the condition when the meditator is in the PS in the Med, but is not detected in any other conditions. These results suggest a possibility that the meditator exerts a peculiar influence on the environment around him, which is transformed by the PS, which then has a delayed effect on the biosensors.

By verifying the two Working Hypotheses, the hypothesis we proposed in the previous paper [2] about the origin of the delayed non-contact effect has been further justified. The hypothesis is as follows: The meditator exerts a peculiar influence on the environment around him, which does not have a direct effect on the bio-sensors. However, this peculiar influence is transformed by the PS, which then has an effect on the bio-sensors.

So far we have not been able to detect in real-time the peculiar effect exerted by a meditator in experiments using cucumbers as the bio-sensors. However, by repeating further experiments, the peculiar effect by the meditator in Med may be detectable. The issues to be further investigated are as follows: changing the experimental variables such as the structural parameters of the PS (e.g., size and the fractal patterns), the meditation method, and the meditator, also taking into consideration the biological clock of the bio-sensors. So many issues remain to be studied.

We would like the discoveries described in this paper to encourage others to look scientifically at the unknown effect of the PS.

This research was done under The Sakamoto Hypertech Project as a joint activity between Aquavision Academy Co., Ltd. (President: Masamichi Sakamoto) and the International Research Institute (Chairman of the Board of Directors: Mikio Yamamoto).

\section{References}

1) O. Takagi, et al., Meditator's non-contact effect on cucumbers. Intl. J. Phys. Sci. 8(15), 647-651(2013). (doi:10.5897/IJPS2012.3800).

2) O. Takagi, et al., Discovery of an anomalous non-contact effect with a pyramidal structure. Intl. J. Sci. 4(5), 4251(2015). (doi:10.18483/ijSci.714).

3) H. Kokubo, M. Yamamoto \& K. Kawano, Evaluation of noncontact healing using biophotons. J. Intl. Soc. Life Info. Sci. 24(2), 320-327(2006).

4) H. Kokubo, O. Takagi \& M. Yamamoto, Development of a gas measurement method with cucumber as a bio-sensor. $J$. Intl. Soc. Life Info. Sci. 27(2), 200-213(2009).

5) H. Kokubo, S. Koyama, O. Takagi, K. Kawano \& M. Yamamoto, Bio-PK detectivity of fluorescence measurement method: Early spring test. J. Intl. Soc. Life Info. Sci. 30(2), 208-216(2012).

6) H. Kokubo \& M. Yamamoto, Controlled healing power and ways of non-contact healing. J. Intl. Soc. Life Info. Sci. 27(1): 90-105(2009)

7) H. Kokubo, O. Takagi, S.Koyama \& M.Yamamoto, Discussion of an approximated equation for special distribution of controlled healing power around a human body. J. Intl. Soc. Life Info. Sci. 29(1), 23-34(2011).

8) M. Sakamoto, Piramiddo Taiken - Kaiji Sareta Kodai no Eichi (Pyramid experience - Wisdom of the indicated ancient times) Tokyo: Heart Publishers, Inc. (2010) [In Japanese].

9) F. H. Atwater, Accessing anomalous states of consciousness with a binaural beat technology. J. Sci. Explor. 11(3), 263274(1997)

10) H. Kokubo, O. Takagi \& S. Koyama, Application of a gas measurement method - Measurement of ki fields and noncontact healing - . J. Intl. Soc. Life Info. Sci. 28(1), 95112(2010)

11) H. Kokubo, Ki or Psi - Anomalous Remote Effects of MindBody System. New York: Nova Science Publishers, Inc. (2015). 